\title{
The Obstacles and Challenges to the Implementation of the Key Performance Indicators System for Sudanese Universities
}

\author{
Adil Yousif \\ University of Science and Technology, Sudan \\ adiluofk@gmail.com
}

\begin{abstract}
In order to evaluate quality management for improving universities status and to meet the international standards, Sudanese Ministry of Higher Education and Scientific Research determined that universities have to improve their administrative management organization according to the Ministry quality Standards and the key performance indicators system. Sudanese higher education institutions use performance indicators to observe their own performance for comparative reasons, to assist the evaluation of university operations, and to present evidence for the ministry quality assurance audits of university teaching and learning quality. The aim of this research is to determine the challenges and obstacles that face the implementation of the key performance indicators system at Sudanese universities. In this research we conducted a survey for the staff at the Sudanese universities as well as interviews with staff and management. The research results from the questionnaires and interviews revealed that the main obstacles that face the implementation of the key performance indicator system are the lack of the quality management culture; most of the universities staff are not aware of the key performance indicator system; the shortage of the financial support for the system; the heavy teaching load for the staff and the lack of operational integration.
\end{abstract}

Keywords: Key Performance Indicator, Education Quality, TQM, Challenges

\section{Introduction}

Universities and institutions worldwide have undergone extensive reform and change over the past thirty years with the plan of enhancing the quality of the higher education. A major characteristic of this has been the drive to come up with systematic proof of effectiveness and efficiency [1]. Performance indicators system has emerged as a scheme used worldwide to manage and evaluate higher education. Performance indicators System has been applied in several countries to improve the quality of higher education[2].

In order to evaluate quality management to improve universities status and to meet the international standards, the Sudanese Ministry of Higher Education and Scientific Research determined that universities have to improve their administrative management organization according to the Ministry quality Standards and the key performance indicators system.

Sudanese higher education institutions use performance indicators to observe their own performance for comparative reasons, to assist the evaluation of university operations, and to present evidence for the ministry quality assurance audits of university teaching and learning quality. The main aim of this study is to identify the obstacles and challenges that prevent the implementation of Key Performance Indicator KPIs in the Sudanese higher education institutes.

The rest of the paper is organized as follows. Section 2 describes a discussion of the key performance indicator system in the literature; this is followed, in Section 3, 
by the related and the relevant works. Section 4 describes the research methods used in this study. We concluded in section 5.

\section{Key Performance Indicators System}

This section throws some lights on the key performance indicator system and the impact of implementing this system.

\subsection{Background}

Universities and institutions worldwide have undergone extensive reform and change over the past thirty years with the plan of enhancing the quality of the higher education. A major characteristic of this has been the drive to come up with systematic proof of effectiveness and efficiency [1]. Performance indicators system has emerged as a scheme used worldwide to manage and evaluate higher education. Performance indicators System has been applied in several countries to improve the quality of higher education[2].

Nevertheless, the related works on performance indicators imply that their implementation may lead to dysfunctional effects. Some authors had voiced their concerns that performance indicators could set the criteria for performance. They argued that the KPI may make academics to direct their effort away from their functional objectives to focus on those considered and funded by the KPI system. These indicators do not always represent the full dimensions of an academic's work performance[2, 3]. Furthermore, not all activities and objectives are able to be quantified, and an weight on easily countable features may make universities to lose main factors of performance indicators [4, 5].

Performance Analysis process examine maintenance plan effectiveness. The differences between actual process performance and the required performance are recognized. In this process a comparison between historical maintenance data the current process performance is conducted. Costs of maintenance process are evaluated. Important performance shortages are handled by revisiting the Work Identification function[6].

The question "Are there any suitable quantitative indicators of performance?" is handled by Guy and Chris (2005). The study stated that "Regarding the classification of the potential indicators, it is necessary to offer an introductory framework to investigate the rate and limit of internationalization"[7].

The studies carried out by the Department of Education, Science and Technology (2001) revealed that the interest in the performance indicators in high education system has been on the rise in some countries, Australia in particular.

\subsection{Impact of Performance Indicators}

Universities consider institutional ranking as significant issue because of the reputation, promotion and publicity related to ranking system. Furthermore, the university ranking would by some means be used to affect the student's selection of university. Professor Brian Wilson, the Chair of the former Committee for Quality Assurance in Australian Higher Education reported that "When we started out, money was the big incentive. But after the first report, there were institutions that would have been quite happy to give the money up if they could have got into Group One"[8].

Moreover, when a university defined the performance indicators for its staff and used these indicators to the university resource allocation process, the university staff will seek out ways to improve their position as measured by the defined indicators; this imply a real improvement in productivity. 
The important part of the application of performance indicator system is giving a mean of fairness to university staff, as the staff knows that the university management is considering their works and value them.

\subsection{KPI System at Sudanese Universities}

In order to evaluate quality management to improve universities status and to meet the international standards, the Sudanese Ministry of Higher Education and Scientific Research determined that universities have to improve their administrative management organization according to the Ministry quality Standards and the key performance indicators system. Sudanese higher education institutions use performance indicators to observe their own performance for comparative reasons, to assist the evaluation of university operations, and to present evidence for the ministry quality assurance audits of university teaching and learning quality. The performance indicators used by the ministry of higher education can be summarized as: for research (publications, external research income, and higher degree research completions) and teaching (student surveys on teaching, graduate employment outcomes, and coursework student completions)[9].

\section{Related Work}

AlTurki, U. and S. Duffuaa [10] presented a study to develop a performance measurement system for educational institutions. This study figured out the properties of adequate performance measures and the steps for developing such measures in an educational environment. The study suggested three types of performance measures: outcomes, inputs and process measures.

The suggested measures aims to choose the most appropriate and relevant performance measures appropriate to academic departments' goals and objectives[10]. The research works presented in[11] shows that the status of science and technology indicators in Sudan. The results of this study revealed that considering standard indicators Sudan lags behind the world's developed and leading developing countries in terms of inputs and outputs indicators. That also holds for the average share of hightechnology exports, GDP per capita grow th, number of scientific publications, level of share in international publication and $\mathrm{n}$ umber of patent filings[11].

Khaleef [12] conducted a study to examine the different effects leading to creativity of traditional and modern education in the Sudan. Four creativity tests, suitably modified for local conditions, were administered to 264 male and female students, with an age range of 15-20 years. The results showed that the groups of students obtaining modern education achieved significantly better than the traditionally educated group on two of the four creativity tests, however with equal performance on one test. The traditionally educated group performed significantly better on the fourth test $[12,13]$.

In Arab countries many studies have been conducted to examine the KPIs system for higher education. The study presented in [14] attempts to examine the challenges of quality management performance in higher education universities in UAE. The researchers conducted a survey at a leading University of Sharjah, using multiple parts questionnaire. The authors in [15] suggested a conceptual framework for assessing IT innovation implementation in higher educational institutions in the developing countries.

The authors in [16]evaluated the level of Quality Management implementation in Qatari private universities. The output of the study is oriented to private and public educational universities; mainly the institute that aim to establish an accreditation within their universities in the Middle East. 
A framework for measuring performance of higher education institution in running its academic, research and supporting functions is presented in the study by Kadarsah Suryadi (2007) as shown in Figure 1[17]. The framework is based on key success factors related to institution sustainability. In their framework key performance indicators are classified into academic, research and supporting key performance indicators (KPI) [17].

KPI Identificationin [17] is based on identifying a list of KPI associated to each criteria. This list is accomplished by experts who are recognizable or concerned in HEI processes. In sequence to be more practical, the selected KPI are examined by experts group in a Delphi Forum [17]. Delphi forum aims to establish the most appropriate and practical KPIs for HEI.

The second phase is the KPI Selection. In this phase, if there are too many KPI, it will be hard to deal with and measure. Therefore, it requires choosing the most essential KPIs that have significant contribution to HEI performance. To group this list in descending order of relevance, the study employed a survey with experts who are directly concerned in HEI activities. The questionnaire, in which the experts have to provide a mark to each indicator, is created. The questionnaire utilized the three-point scale of "not important", "somewhat important" and "very important" using "Cut off Point" approach as proposed by Tam et al[18]. . In Tam et al[18] result is the selected KPIs based on its level of significance [17]. 


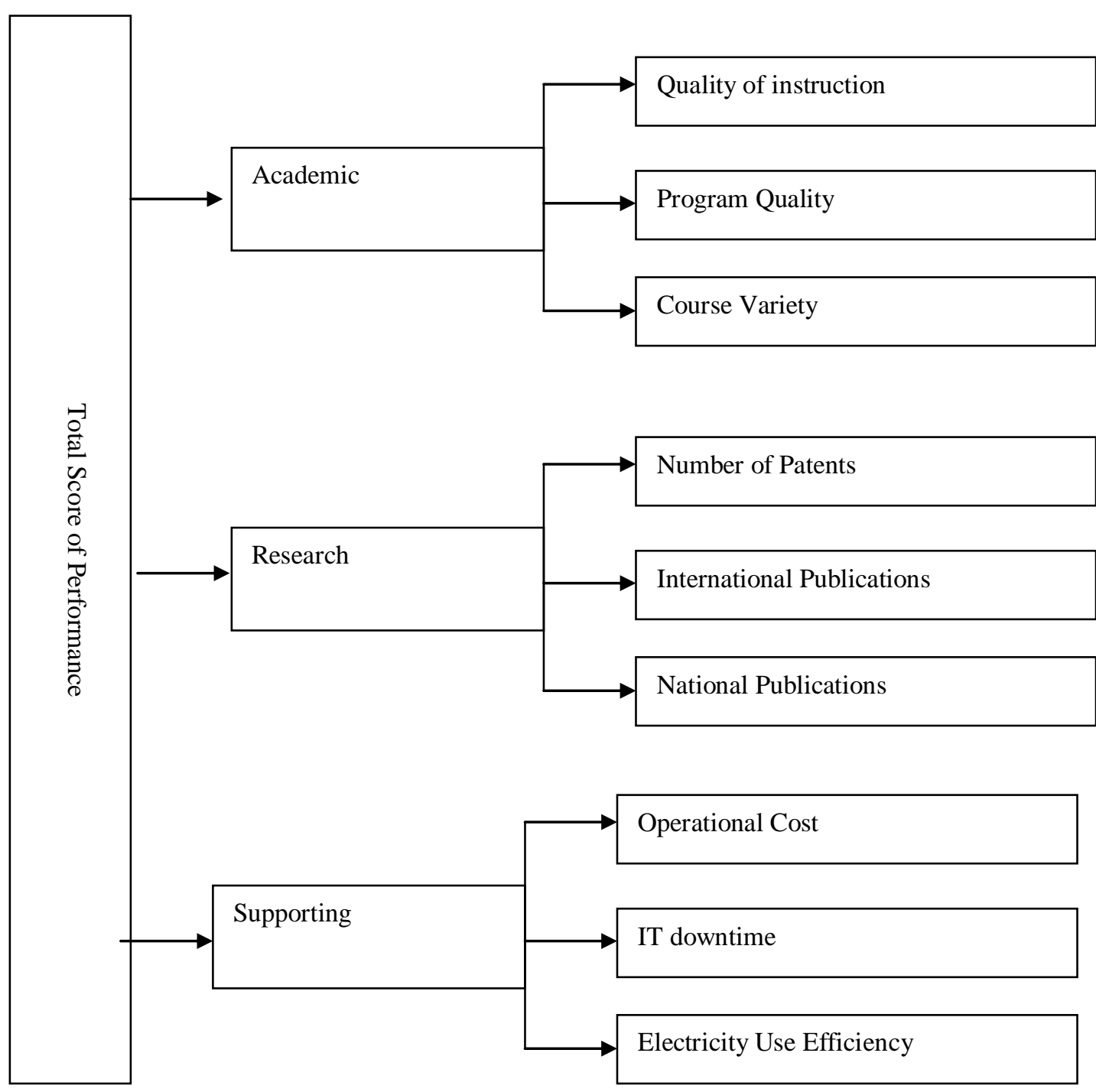

Figure 1. KPI Framework based on Analytic Hierarchy Process (AHP) [17]

\section{The Research Methodology}

The purpose of this research study is to facilitate a more in-depth investigation of to what extends that Sudanese universities are implementing KPIs, and what are the obstacles and challenges to the implementation of the Key Performance Indicator System for Sudanese universities. Furthermore, this study aims to find out whether any actions or supports are needed. This research study conducted a survey to collect information about the application of performance indicator at Sudanese universities.

\subsection{Sample and Data Collection}

This research study conducted a survey for the staff and management members of a number of Sudanese universities. Furthermore, qualitative research and interviews were employed with the Sudanese university's senior executive and quality 
manager. The research population has been selected using standard sampling methodology. A questionnaire is prepared that consists of several questions related to the study. The first part of the questionnaire identify to what extend that the university apply the KPI system. The second part is formulated to find out the main obstacles and challenges facing the implementation of the KPI system.

Six representative Sudanese universities were selected for the survey. They consist of two public universities in Khartoum, capital of Sudan, a public university at far state from Khartoum, a private university, and private college and Islamic university. The disciplines from which the academics were selected for participation covered humanities, science, and professional studies. Within each discipline and each level, the university academics who were interviewed were randomly chosen. The interview covered the questionnaire and six interview statements, which are similar to those in the questionnaire but considered at a more in-depth level. The remaining staff in this study completed a questionnaire. Participation was voluntary, and the Participants were assured of confidentiality of results.

The universities which participated in the project were:, Sudan university of Science and Technology, Alnilain University, Kassala University, Quran Kareem University, Garden City College and University of Science and Technology.

\subsection{Analysis}

Data from the questionnaires were analyzed by means of Statistical Package for Social Sciences and Microsoft Excel.

\subsection{The Results and Discussions}

The following subsections describe the results of the study.

\subsubsection{The Questionnaires}

After distributing the questionnaires and collecting and summarizing the data, the following observations and results are obtained.

The main results of the study aims to find out the critical factors that affects the implementations of key performance system for the Sudanese universities and institutes.

The data shown in Table 1 and Figure 2 is based on the questionnaire only. The data collected from the interviews are discussed in section 4.3.2.

Table 1. The Obstacles for Implementing KPIs in Sudanese Universities

\begin{tabular}{|l|c|}
\hline The Obstacles & Frequency \\
\hline The lack of the quality management culture & 33 \\
\hline $\begin{array}{l}\text { Some of universities staff are not aware of the key } \\
\text { performance indicator system }\end{array}$ & 27 \\
\hline The shortage of the financial support for the system & 21 \\
\hline The heavy teaching load for the staff & 18 \\
\hline The lack of operational integration & \\
\hline
\end{tabular}




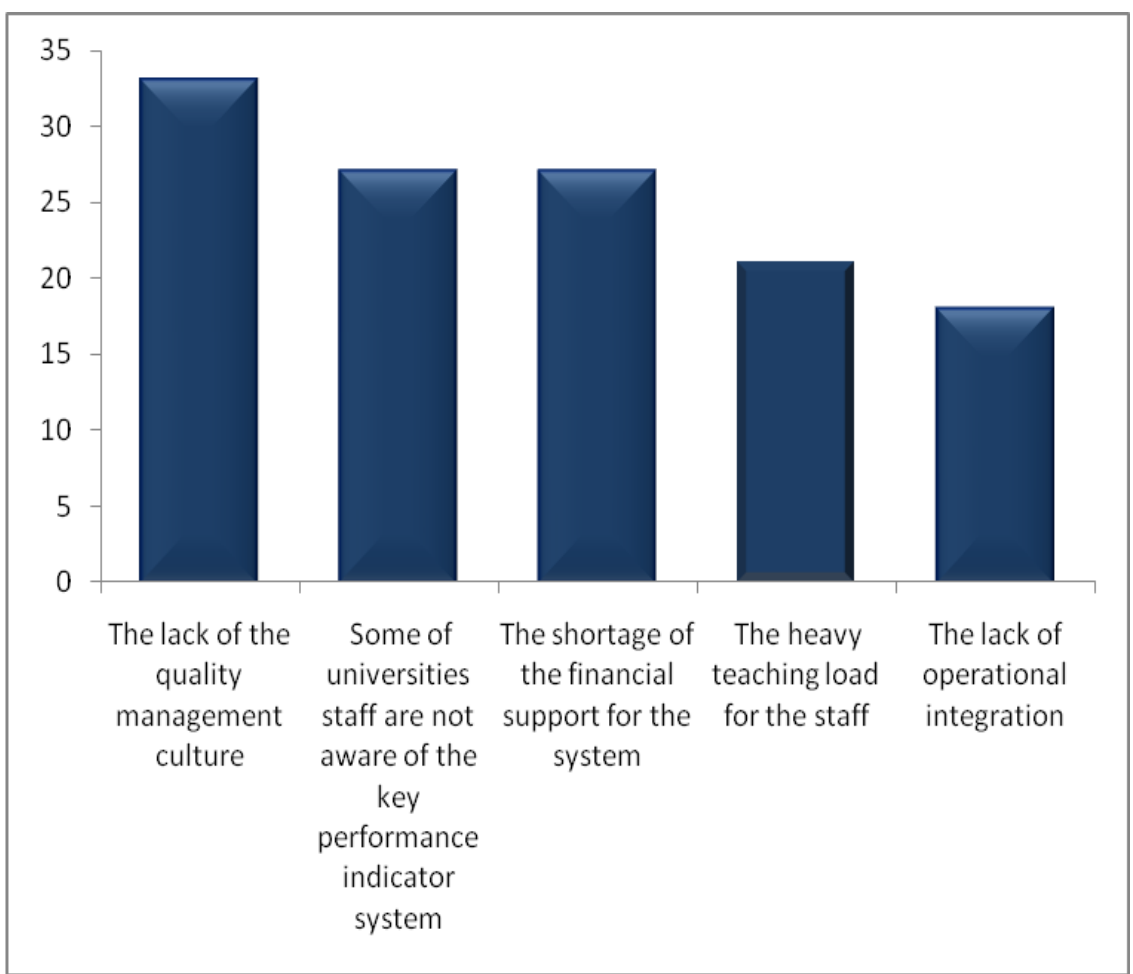

Figure 2. The Obstacles for Implementing KPIs in Sudanese Universities

As described in Table 1 and Figure 2, most of the participants reported there is a lack of the quality management culture and KPIs system as most of the staff are not aware of the KPIs standard presented by the ministry of higher education.

As shown in Table 1 and Figure 2, a majority of the participants also mentioned that the financial support for the KPIs system; as the system needs full time staff, offices, computers and other tools.

Furthermore, several participants reported that the heavy load for the university staff prevent them from implementing and improving the performance factors. Relating to the heavy load with the financial shortage the staff sometimes needs to work in more than one university to increase their income in order to face the living expensive. Finally, some staff mentioned the lack of operation integration as an important obstacle for implementing the KPIs system.

\subsubsection{The Interviews}

The interviews with staff and management selected by the study revealed that there are several obstacles that face the implementation of quality management and KPIs system such as the lack of quality culture, the shortage of financial support for the KPIs system. Some participants in the study reported that the ministry of higher education is not serious about implementing the KPIs system; this because the ministry have the power and the authority to force the institutes and universities to implement the KPIs system but it does not. They declared the ministry of higher education can set a rule that each university that does not apply the KPIs system the ministry will not send students to that institute or at least decrease the numbers of students. 


\section{The Conclusion and Recommendations}

The purpose of this research study was to facilitate a more in-depth investigation of to which extends that Sudanese universities are implementing KPIs. Furthermore the study aims to find out what are the obstacles and challenges to the implementation of the Key Performance Indicator System for Sudanese Universities and whether any actions or supports are needed. The most significant results from this research study are:

- The key challenges and obstacles facing the implementation of Key Performance Indicator System at Sudanese University are:

i. The lack of the quality management culture.

ii. Some of universities staff are not aware of the key performance indicator system.

iii. The shortage of the financial support for the system.

iv. The heavy teaching load for the staff.

- The lack of operational integration.

To help in tackling and handling the obstacles and challenges presented in this paper, the study proposes the following recommendations:

- Encourage a culture of quality.

- Pay more consideration to incentives for university staff.

- Support and fund the scientific research.

- Appoint managements that have knowledge in implementation of Quality Management and KPIs system.

\section{References}

[1] J. Guthrie and R. Neumann, "Performance Indicators in Universities: The Case of the Australian University System", Submission for Public Management Review Final February, (2006).

[2] J. Taylor, "The impact of performance indicators on the work of university academics: evidence from Australian universities", Higher education quarterly, vol. 55, (2001), pp. 42-61.

[3] G. Johnes, "Performance indicators in higher education: a survey of recent work", Oxford Review of Economic Policy, (1992), pp. 19-34.

[4] F. J. R. C. Dochy, M. S. R. Segers and W. H. F. W. Wijnen, " Management Information and Performance Indicators in Higher Education: An International Issue", Assen, Netherlands: Van Gorcum., (1990).

[5] P. Ramsden, "A performance indicator of teaching quality in higher education: The Course Experience Questionnaire", Studies in higher education, vol. 16, (1991), pp. 129-150.

[6] A. Weber and I. R. Thomas, "Key performance indicators", Measuring and Managing the Maintenance Function, Ivara, (2005).

[7] G. Carrin and M. C. James, "Key performance indicators for the implementation of social health insurance", Applied Health Economics and Health Policy, vol. 4, (2005), pp. 15-22.

[8] G. Maslen, "At least the teaching has improved", Campus Review, vol. 5, (1995), p. 6.

[9] H. Education, "Guide national standards to ensure the quality of higher education in Sudan", (2010).

[10] U. Al-Turki and S. Duffuaa, "Performance measures for academic departments", International Journal of Educational Management, vol. 17, (2003), pp. 330-338.

[11] S. S. O. M. Nour, "Assessment of Science and Technology Indicators in Sudan", Science Technology \& Society, (2012), pp. 323-354.

[12] O. H. Khaleefa, G. Erdos and I. H. Ashria, "Traditional Education and Creativity in an Afro-Arab. Islamic Culture: The Case of Sudan", The Journal of Creative Behavior, vol. 31, (1997), pp. 201-211.

[13] O. Ugwu and T. Haupt, "Key performance indicators and assessment methods for infrastructure sustainability - a South African construction industry perspective", Building and Environment, vol. 42, (2007), pp. 665-680.

[14] A. M. M. Ahmed and B. I. Hamdoon, "The Challenges and obstacles of TQM Implementation in the Higher Education Institutions: The Case of Sharjah University in UAE", (2007).

[15] M. Q. Huda and H. Hussin, "A conceptual model of information technology innovation implementation effectiveness in higher education", Information and Communication Technology for the Muslim World (ICT4M), 5th International Conference, (2013). 
[16] N. F. Sulaiman, N.-N. Manochehri and R. A. Al-Esmail, "Level of Total Quality Management Adoption in Qatari Educational Institutions: Private and Semi-Government Sector", Journal of Education for Business, vol. 88, (2013), pp. 76-87.

[17] K. Suryadi, "Framework of measuring key performance indicators for decision support in higher education institution", Journal of Applied Sciences Research, vol. 3, (2007), pp. 1689-1695.

[18] M. C. Tam and V. R. Tummala, "An application of the AHP in vendor selection of a telecommunications system", Omega, vol. 29, (2001), pp. 171-182.

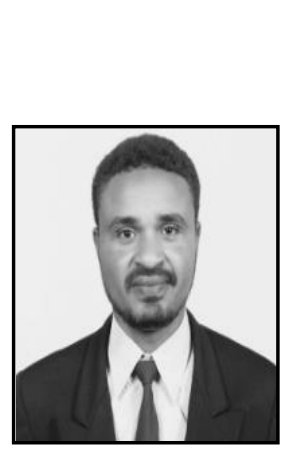

\section{Author}

Adil Yousif received the B.Sc. and M.Sc. from University of Khartoum, Sudan, in 2003 and 200 respectively. He obtained his $\mathrm{PhD}$ degree from UTM, University of Technology in Malaysia in 2013. $\mathrm{He}$ is now Assistant Professor at Faculty of Computer Science and Information Technology, University of Science and Technology. He is also the postgraduate study coordinator at faculty of computer science and Information technology, University of Science and Technology - Sudan. He is also a member of PCRG research group. He has published more than ten papers in international journals and conferences. His research interests include computer networks, cloud computing, grid computing and optimization techniques. He has some researches and seminars on the higher education quality and teaching methods. 
International Journal of Education and Learning

Vol.6, No.1 (2017) 